Trauma Berufskrankh 2010 - 12 [Suppl 1]:78-85 DOI 10.1007/s10039-010-1601-3

Online publiziert: 6. Februar 2010

(c) Springer-Verlag 2010

\author{
M.J. Strobel ${ }^{1,2}$ \\ ${ }^{1}$ Sporthopaedicum Straubing \\ ${ }^{2}$ ATOS Privatklinik, München
}

\title{
Arthroskopische Eingriffe am Kniegelenk
}

\section{Wann ambulant - Wann stationär?}

Ambulante arthroskopische Eingriffe am Kniegelenk sind populär, da sie den $\mathrm{Pa}$ tienten wenig belasten. Zudem fühlt sich dieser bei einem ambulanten Eingriff nicht so krank wie bei einer stationären Aufnahme für mehrere Tage, v. a. wenn er dort z. B. bei der Aufnahme gefragt wird: „Wen sollen wir beim Auftreten von Komplikationen benachrichtigen?" Nicht zuletzt auch aus Kostengründen wird der ambulante Eingriff von den Kostenträgern favorisiert, manche Patienten werden sogar dazu gedrängt, sich nur ambulant operieren zu lassen.

Dennoch gilt es sowohl für den operativ tätigen Arzt als auch für den Patienten, die ambulante Operation sorgfältig abzuwägen. Hierbei ist nicht nur die Indikation als Kriterium heranzuziehen, sondern es sind v. a. die sozialen Bedingungen, in denen der Patient lebt, als wichtiges, manchmal sogar limitierendes Entscheidungskriterium zu beachten.

\section{Definition und Qualifikation}

Nach den Richtlinien der Bundesärztekammer zur Qualitätssicherung wird die ambulante Operation wie folgt definiert: Die ambulante Operation ist dadurch gekennzeichnet, dass der Patient im Allgemeinen die Nacht vor und nach dem Eingriff zu Hause verbringt. Ausgenommen sind sog. Kleineingriffe, die in Art und Behandlungsumfang im Benehmen mit den medizinisch wissenschaftlichen Fachgesellschaften und Berufsverbänden zu regeln sind.
Es ist sicherzustellen, dass der Patient durch den ambulant durchgeführten Eingriff während der prä-, peri- und postoperativen Behandlung keinem höheren Risiko (auch in Zusammenhang mit der postoperativen Betreuung) ausgesetzt sein darf als bei einer Behandlung unter stationären Bedingungen.

Die fachspezifischen Anforderungen zur Qualitätssicherung ambulanter Operationen werden in den Anlagen zu dieser Richtlinie geregelt. Diese Anlagen wurden von der Bundesärztekammer in Zusammenarbeit mit den jeweils zusätzlichen medizinisch-wissenschaftlichen Fachgesellschaften und Berufsverbänden erstellt:

Die persönliche Qualifikation der ambulant operierenden Ärzte bzw. der Anästhesisten entspricht dem Facharztstandard. Der Facharztstandard setzt ausreichende Kenntnisse, Fähigkeiten und Fertigkeiten in der Notfallmedizin und der Beherrschung prä-, peri- und postoperativer Komplikationen in Zusammenhang mit der durchgeführten Operation bzw. Anästhesie voraus.

Der Operateur hat zudem dafür Sorge $\mathrm{zu}$ tragen, dass gegebenenfalls beteiligtes Assistenzpersonal in ausreichender Zahl mit einer ausreichenden Qualifikation zur Verfügung steht.

Demnach werden sowohl hinsichtlich der Ziele der ambulanten Operation als auch der Qualität hohe Anforderungen an den Operateur, den Anästhesisten und das Assistenzpersonal gestellt.

\section{Räumliche Anforderungen}

Ambulante Operationen können im Krankenhaus, in der Arztpraxis oder in speziell darauf ausgerichteten Ambulatorien durchgeführt werden.

\section{Ambulante Operationen im Krankenhaus}

Ambulante Operationen lassen sich bekanntlich nicht in den normalen Alltag eines Krankenhauses integrieren. Das „Denken“ im Krankenhaus ist zudem dadurch geprägt, dass der Tagesablauf durch die Arbeitszeiten des Personals bzw. die Arbeitszeiten und Motivation der Anästhesisten begrenzt bzw. limitiert wird. $\mathrm{Zu}$ dem ist zu bedenken, dass es sich bei ambulanten Operationen um kleinere Eingriffe handelt, gleichzeitig aber das Operationspersonal hinsichtlich der Wechsel- und Reinigungszeiten des Operationssaales (OP) auf größere Operationen eingestellt ist. Hinzukommt, dass die Entlohnung des gesamten Operationspersonals nicht in Abhängigkeit der geleisteten Arbeits- bzw. durchgeführten Operationszahl erfolgt, sondern vom Krankenhausträger in der Tarifstruktur (öffentlicher Dienst) vorgegeben wird. Eine Mehrarbeit, wie sie z. B. bei ambulanten Operationen notwendig ist - hier kümmert sich das Operationspersonal auch um die Vorund Nachbetreuung der ambulanten Patienten, ist im Krankenhaus keinesfalls vorgesehen. 
Hier sind neue Stellen und neue Räumlichkeiten zu schaffen, um z. B. einen Einschleusungsbereich, einen Aufwachraum und einen Ausschleusungsbereich zu bieten. Auch die interne Logistik des Operationsteams mit Erstellen des Operationsberichtes unmittelbar nach der Operation muss im Gegensatz zum normalen stationären Ablauf komplett geändert werden. Zum Entlassungszeitpunkt sollte der ambulant operierte Patient sein Operationsprotokoll bzw. den Operationsbericht einschließlich der zu erfolgenden Nachbehandlung erhalten. Zudem müssen ihm z. B. Rezepte, Medikamente, Gehstützen oder sonstige orthopädische Hilfsmittel ausgehändigt bzw. zur Verfügung gestellt werden. Dieser gesamte Ablauf erfordert ein völliges Umdenken und Umstrukturieren im Vergleich zum stationären Vorgehen. Der Operateur sollte nach der Operation mit dem Patienten immer das weitere Vorgehen in einem persönlichen Gespräch erklären. Auch dies steht im deutlichen Unterschied zum normalen stationären Behandlungsablauf.

\section{Ambulante Operationen in der Praxis}

Viele niedergelassene Kollegen sind dazu übergangen, ambulante Operationen in ihrer Praxis durchzuführen. Hierzu werden 1 oder 2 Praxisräume umgestaltet, ohne dabei jedoch die strengen Qualitätskriterien, die an ein effizientes ambulantes Operieren zu stellen sind, komplett erfüllen zu können. Es wird somit vielfach mit Kompromissen hinsichtlich der räumlichen Abtrennung des Ein- und Ausschleusebereichs sowie des Aufwachraumes gearbeitet. Diese Kompromisse sind notwendig, da viele Praxen primär nicht auf die Anforderungen des ambulanten Operierens konzipiert wurden.

\section{Ambulante Operationen im Ambulatorium}

In den letzten Jahren eröffneten zahlreiche ambulante Operationszentren in Deutschland. Diese wurden meist von Anästhesisten geplant und organisiert und werden in Zusammenarbeit mit operierenden Fachkollegen (Orthopäden, Chirurgen, HNOÄrzten, Gynäkologen, Augenärzten, Uro-

Trauma Berufskrankh 2010 - 12 [Suppl 1]:78-85 DOI 10.1007/s10039-010-1601-3

C) Springer-Verlag 2010

M.J. Strobel

Arthroskopische Eingriffe am Kniegelenk. Wann ambulant - Wann stationär?

\section{Zusammenfassung}

Ambulante arthroskopische Eingriffe am Kniegelenk belasten den Patienten nur wenig und werden aus finanziellen Gründen auch von den Kostenträgern favorisiert. Dennoch muss vor Durchführung einer ambulanten Operation eine sorgfältige Abwägung erfolgen, wobei nicht nur die Indikation als Kriterium heranzuziehen ist, sondern v. a. die sozialen Lebensbedingungen des Patienten entscheidend sind. Von der Bundesärztekammer werden sowohl hinsichtlich der Ziele der ambulanten Operation als auch der Qualität hohe Anforderungen an den Operateur, den Anästhe- sisten und das Assistenzpersonal gestellt. Die Eingriffe können im Krankenhaus, in der Arztpraxis oder in speziell darauf ausgerichteten Ambulatorien erfolgen. Im vorliegenden Beitrag werden u. a. das am Sporthopaedicum Straubing etablierte interne Organogramm für ambulante Operationen, Fehlermöglichkeiten sowie Entscheidungskriterien bezüglich der ambulanten vs. stationären Durchführung vorgestellt.

\section{Schlüsselwörter}

Kniegelenk · Arthroskopie · Ambulante

Operation · Anforderungen $\cdot$ Ablauf

\section{Knee arthroscopy. Deciding between an outpatient and an inpatient procedure?}

\section{Abstract}

Outpatient arthroscopic knee interventions are well tolerated by the patient and, for cost reasons, are also favoured by the cost-bearers. Nevertheless, carrying out outpatient surgery requires careful consideration not only of the indication as the sole criterion, but more importantly of the patient's social/living conditions. The German medical association puts high demands on the operator, the anesthetist and on the assisting personnel not only in terms of the quality, but also of the goals of outpatient surgery. Interven- tions can be carried out in hospitals, medical practices or in dedicated outpatient clinics. The current article discusses the internal organogram for outpatient surgery established at the Sporthopaedicum in Straubing, Germany, potential errors, as well as decisionmaking criterion regarding outpatient vs. inpatient surgery.

\section{Keywords}

Knee joint · Arthroscopy · Outpatient surgery . Demands · Procedure 


\begin{tabular}{|c|c|c|c|}
\hline $\begin{array}{l}\text { OPS- } \\
\text { Ziffer }\end{array}$ & $\begin{array}{l}\text { An- } \\
\text { zahl }\end{array}$ & $\begin{array}{l}\text { An- } \\
\text { teil } \\
(\%)\end{array}$ & Prozedur \\
\hline $5-812$ & 4195 & 16 & Arthroskopie \\
\hline $5-056$ & 3061 & 11 & Neurolyse \\
\hline $5-530$ & 2353 & 9 & Leistenbruch \\
\hline $5-385$ & 1803 & 7 & Varizenoperation \\
\hline $5-787$ & 1352 & 5 & Metallentfernung \\
\hline $5-840$ & 1220 & 5 & $\begin{array}{l}\text { Operationen an } \\
\text { Sehnen der Hand }\end{array}$ \\
\hline $5-895$ & 1127 & 4 & Hauttumor \\
\hline $5-849$ & 823 & 3 & $\begin{array}{l}\text { Operationen an der } \\
\text { Hand }\end{array}$ \\
\hline $5-813$ & 811 & 3 & $\begin{array}{l}\text { Arthroskopie des } \\
\text { Kapsel-Band-Apparats }\end{array}$ \\
\hline $5-788$ & 612 & 2 & Operationen am Fuß \\
\hline Rest & 9463 & 35 & \\
\hline \multicolumn{4}{|c|}{$\begin{array}{l}\text { OPS Operationen- und Prozedurenschlüssel, } \\
n=26.820 \text { Fragebögen }\end{array}$} \\
\hline
\end{tabular}

Tab. 3 Operationen Sporthopaedicum Straubing (1992-2007)

\begin{tabular}{lll} 
Operationsart & $\begin{array}{l}\text { Ge- } \\
\text { samt }\end{array}$ & $\begin{array}{l}\text { Am- } \\
\text { bulant }\end{array}$ \\
Arthroskopie Knie & 35.957 & 21.438 \\
\hline $\begin{array}{l}\text { VKB-Rekonstruktion } \\
12.706\end{array}$ & 4661 \\
\hline HKB-Rekonstruktion & 979 & 79 \\
\hline $\begin{array}{l}\text { Arthroskopie der Schulter } \\
\text { Arthroskopie anderer }\end{array}$ & 7488 & 1156 \\
$\begin{array}{l}\text { Gelenke } \\
\text { HKB hinteres Kreuzband, VKB vorderes Kreuzband }\end{array}$ \\
\hline
\end{tabular}

Tab. 5 Ablauforganisation für ambu-

lante Operationen am Sporthopaedicum Straubing

1. Aufnahme

2. Prämedikation

3. Patientenvorbereitung

4. Einleitung

5. Operationssaal

6. Dokumentationsbereich

7. Aufwachraum

8. Patienteninformation

9. Entlassung und Ausschleusung

logen usw.) betrieben. Oft mietet der Operateur einen Operationssaal und zahlt für jede durchgeführte Operation oder je nach Operationszeit eine Nutzungsgebühr an den Besitzer bzw. Betreiber des Ambulatoriums.
Tab. 2 Häufigste Eingriffe nach OPS-

Ziffern in der Orthopädie

OPS- An- Anteil Prozedur

Ziffer zahl (\%)

5-812 $11.415 \quad 49 \quad$ Arthroskopie

5-813 21569 Arthroskopie des Kapsel-BandApparats

\begin{tabular}{|llll|}
\hline $5-056$ & 1809 & 8 & Neurolyse \\
\hline $5-814$ & 1232 & 5 & $\begin{array}{l}\text { Arthroskopie der } \\
\text { Schulter }\end{array}$ \\
\hline
\end{tabular}

5-810 $796 \quad 3 \quad$ Gelenkrevisions-

\begin{tabular}{llll} 
& & & arthroskopie \\
\hline $5-788$ & 583 & 3 & Operation am Fuß \\
\hline $5-830$ & 567 & 2 & Wirbelsaulen-
\end{tabular}

5-830 $567 \quad 2 \quad$ Wirbelsäulen-

\begin{tabular}{|cccl}
\hline $5-787$ & 559 & 2 & Metallentfernung \\
\hline $5-811$ & 541 & 2 & $\begin{array}{l}\text { Synovialisarthro- } \\
\text { skopie }\end{array}$ \\
\hline $1-697$ & 535 & 2 & $\begin{array}{l}\text { Diagnostische } \\
\text { Arthroskopie }\end{array}$ \\
\hline
\end{tabular}

Rest $2992 \quad 13$

OPS Operationen- und Prozedurenschlüssel, $\mathrm{n}=23.185$ Fragebögen

\begin{tabular}{|c|c|c|}
\hline $\begin{array}{l}\text { OPS- } \\
\text { Ziffer }\end{array}$ & $\begin{array}{l}\text { Anteil } \\
\text { (\%) }\end{array}$ & Prozedur \\
\hline $5-814$ & 12,56 & Arthroskopie der Schulter \\
\hline $5-788$ & 2,04 & Operation am Fuß \\
\hline $1-697$ & 0,79 & $\begin{array}{l}\text { Diagnostische Arthro- } \\
\text { skopie }\end{array}$ \\
\hline $5-813$ & 0,77 & $\begin{array}{l}\text { Arthroskopie des Kapsel- } \\
\text { Band-Apparats }\end{array}$ \\
\hline $5-811$ & 0,59 & Synovialisarthroskopie \\
\hline $5-812$ & 0,40 & Arthroskopie \\
\hline $5-787$ & 0,19 & Metallentfernung \\
\hline $5-056$ & 0,12 & Neurolyse \\
\hline $5-810$ & 0,00 & $\begin{array}{l}\text { Gelenkrevisionsarthro- } \\
\text { skopie }\end{array}$ \\
\hline $5-830$ & 0,00 & $\begin{array}{l}\text { Arthroskopie an der } \\
\text { Wirbelsäule }\end{array}$ \\
\hline \multicolumn{3}{|c|}{$\begin{array}{l}\text { OPS Operationen- und Prozedurenschlüssel, } \\
\text { n=19.055 Fragebögen }\end{array}$} \\
\hline
\end{tabular}

\section{Anforderungen}

Da im ambulanten Bereich - und dieser ist meist privatwirtschaftlich organisiert ein wesentliches Augenmerk auf die Ablauforganisation zu richten ist, muss dies schon bei der Planung beachtet werden. Somit sind im ambulanten Operationszentrum ein Empfangsbereich für die Patienten und deren Angehörige, Aufent- haltsräume für die Angehörigen, aber auch ein Einschleusebereich mit mindestens 2, besser 3 Schleusen erforderlich. Da die Patienten vom Anästhesisten vor dem Eingriff prämediziert werden, falls dies noch nicht zuvor erfolgt ist, müssen hierfür ebenfalls Räumlichkeiten zur Verfügung stehen. Auch muss es möglich sein, fehlende Blutwerte, die der Patient möglicherweise nicht parat hat oder die kontrolliert werden müssen, erneut zu bestimmen.

Im Operationsbereich ist ein spezieller Einleitungsbereich für die Narkose erforderlich.

Je mehr ambulante Operationen durchgeführt werden, desto größer muss der Aufwachraum konzipiert werden, da es nicht dazu kommen darf, dass Patienten, die sich nach dem Eingriff noch nicht ausreichend erholt haben, den Aufwachraum verlassen müssen, weil die nächsten 2 oder 3 Patienten darauf warten, in diesen verbracht zu werden. Hat der Patient ein ausreichendes Vigilanzniveau erreicht, kann er den Aufwachraum verlassen. Zuvor erfolgen ein Gespräch mit dem Operateur und die Übergabe des Operationsprotokolls an den Patienten. Zudem muss sich der Anästhesist vom ausreichenden Vigilanzniveau überzeugen.

Im Ausschleusebereich sollte dem Patienten noch einmal von einer Schwester oder einem Mitarbeiter des Ambulatoriums erklärt werden, wie er sich nach der Operation zu verhalten hat. Schmerzmedikamente und Thrombosespritzen für die ersten Tage werden ihm mitgegeben, und er wird unterwiesen, wie er diese Medikamente zu handhaben hat. Es ist hilfreich, wenn bei diesem Abschlussgespräch die Angehörigen zugegen sind, da der Patient in der Aufregung oft das Wesentliche nicht ganz aufnimmt bzw. behalten kann. Hier ist es wichtig, dass die notwendigen Nachbehandlungsmaßnahmen sowie Verhaltensregeln für den Notfall dem Patienten und seinen Angehörigen in schriftlicher Form mitgegeben werden.

Es ist darauf zu achten, dass der Patient immer von seinen Angehörigen abgeholt und nach Hause transportiert wird und nicht von einem entfernten Freund oder einem Taxifahrer. Sollte es während des Transports zu einer unvorhergesehenen Situation kommen, kümmern sich An- 
gehörige intensiver um den Patienten als entfernte Bekannte oder ein unbekannter Fahrer.

Demnach ist festzustellen, dass die Erfordernisse an ein ambulantes Operationszentrum extrem hoch sind und eine präzise, geplante Ablauforganisation vorhanden sein muss, um das ambulante Operieren sowohl für den Patienten als auch den Operateur effizient und sicher und gleichzeitig komplikationsarm zu gestalten.

\section{Wirtschaftliche Gesichtspunkte}

Operateure bzw. Anästhesisten gehen ein erhebliches wirtschaftliches Risiko ein, wenn sie ein komplett ausgestattetes ambulantes Operationszentrum betreiben. Staatliche Zuschüsse wie sie z. B. Krankenhäuser oder Universitätskliniken erhalten, sind nicht zu erwarten. Dieses mit der großen Investition für ein ambulantes Operationszentrum einhergehende beträchtliche wirtschaftliche Risikopotenzial für den Betreiber des Ambulatoriums darf nicht unberücksichtigt bleiben, wenn das ambulante Operieren im Ambulatorium mit dem im Krankenhaus verglichen wird. Denn kommt es im Krankenhaus zu Planungsfehlern durch den Anästhesisten, den Operateur oder zu einem sehr langsamen Operationsablauf, hat der Krankenhausträger (Stadt, Land oder Ähnliches) das Risiko bzw. die Kosten zu tragen.

Da ein ambulantes Operationszentrum nur dann wirtschaftlich sinnvoll funktioniert, wenn spezielle Operationen sehr häufig durchgeführt werden, werden gerade in diesen Fällen besonders hohe Investitionskosten hinsichtlich der Operationsinstrumente erforderlich. Im Gegensatz zum Krankenhaus oder Klinikum muss der Betreiber des Ambulatoriums bzw. der Operateur selbst für die Kosten des Operationsinstrumentariums aufkommen. In dem von uns genutzten Ambulatorium (Ambulantes Operationszentrum Straubing) hat das von uns beschaffte Instrumentarium, das erforderlich ist, um einen adäquaten und reibungslosen, gleichzeitig aber auch effizienten Operationsbetrieb zu gewährleisten, laut Kostenanalyse einen Wert von über 850.000,- EUR.

\section{Häufigkeit}

Arthroskopien zählen zu den häufigsten ambulant durchgeführten Eingriffen in der Chirurgie (• Tab. 1). In der Orthopädie ist ihr Anteil noch größer (• Tab. 2).

Die eigenen Erfahrungen im Sporthopaedicum Straubing erstrecken sich über den Zeitraum von 1992-2008, in dem insgesamt 62.950 Operationen durchgeführt wurden. Davon erfolgten 29.253 Eingriffe ambulant (47\%). Der Prozentsatz der ambulanten Operationen liegt je nach Eingriffsarzt und Gelenk zwischen 60\% („normale“ Kniearthroskopie) bis zu unter 10\% (komplexe Bandrekonstruktionen mit Beteiligung mehrerer Bänder oder Eingriffen am hinteren Kreuzband)

\section{(• Tab. 3).}

Eine Analyse von 19.055 beantworteten Fragebögen (AQS1-Studie) bezüglich der zu einer stationären Aufnahme führenden Komplikationen nach ambulanten Operationen ergab, dass hierfür insbesondere Schulterarthroskopien verantwortlich waren (• Tab. 4).

\section{Ablauforganisation}

Sie orientiert sich bei ambulanten Operationen in unserer Einrichtung an einem internen Organogramm, das verschiedene Punkte und Stationen umfasst (• Tab. 5).

Aufnahme. Bei der Patientenaufnahme werden die Personalien des Patienten registriert, und es wird kontrolliert, ob sämtliche präoperative Vorbereitungen (Blutwerte usw.) erfolgt sind. Zudem wird geklärt, ob der Patient in Begleitung ist und von Angehörigen nach der Operation abgeholt werden kann.

Prämedikation. Falls sie nicht bereits einige Tage zuvor vorgenommen wurde, erfolgt die Prämedikation im Anschluss an die Aufnahme durch den Anästhesisten. Es wird erneut überprüft, ob alle Blutwerte in Ordnung sind bzw. die übliche Narkosevorbereitung erfolgt ist. Zudem wird nach dem Nüchternheitsstatus des Patienten gefragt.

Patientenvorbereitung und Einleitung. Der Patient wird in die Patienten- schleuse geleitet. Hier entkleidet er sich bis auf die Unterhose und erhält ein Operationshemd. Er wird noch einmal nach der zu operierenden Seite gefragt, und diese wird mit einem wasserfesten Filzstift im Fußbereich markiert (• Abb. 1). Diese Seitenzuordnung wird noch einmal mit dem Operationsplan verglichen, um Seitenverwechslungen sicher auszuschließen.

Der Kniebereich wird rasiert, und der Patient erhält die erste Antithrombosespritze. Gleichzeitig wird ihm erklärt, wie diese Spritzen nach der Operation zu handhaben sind.

Vor dem Eingriff untersucht der Operateur den Patienten immer noch einmal und überprüft die Diagnose. Sollten sich zu diesem Zeitpunkt Hautveränderungen zeigen - die Arthroskopie ist ein Wahleingriff - oder Zweifel an der Diagnose bestehen, sollte die Operation aufgeschoben werden, bis die Hautveränderungen abgeklungen sind bzw. die Diagnose eindeutig gesichert ist.

Der Patient wird auf den Operationstisch gelegt. Seitlich des zu operierenden Beins wird eine Seitenstütze angebracht, um das Gelenk aufklappen zu können. Einen speziellen Beinhalter mit integrierter Druckmanschette verwenden wir nicht, da dies die Beweglichkeit des Beins deutlich einschränkt und das Anlegen gleichzeitig unnötig Zeit in Anspruch nimmt. Die angelegte Druckmanschette wird wasserdicht abgeklebt. Gleichzeitig wird eine Neutralelektrode auf den Bauch des Patienten aufgeklebt. Dieser erhält einen venösen Zugang, und es erfolgt die Narkoseeinleitung. Die meisten arthroskopischen Operationen werden in Vollnarkose durchgeführt, sofern keine Kontraindikationen hierfür bestehen.

Operative Versorgung. Der Patient wird zur Operation in den Operationssaal gefahren. Nach dem Wundverschluss der Arthroskopiezugänge mit 2 Einzelknopfnähten wird ein elastokompressiver Verband angelegt.

Theoretisch könnte auf das Zunähen der Zugänge nach einer Arthroskopie verzichtet werden. Die Naht verhindert jedoch nicht nur, dass es zu einer Wunddehiszenz kommt, sondern zwingt den Patienten geradezu dazu, den Hausarzt oder 


\section{Arthroskopische Eingriffe}
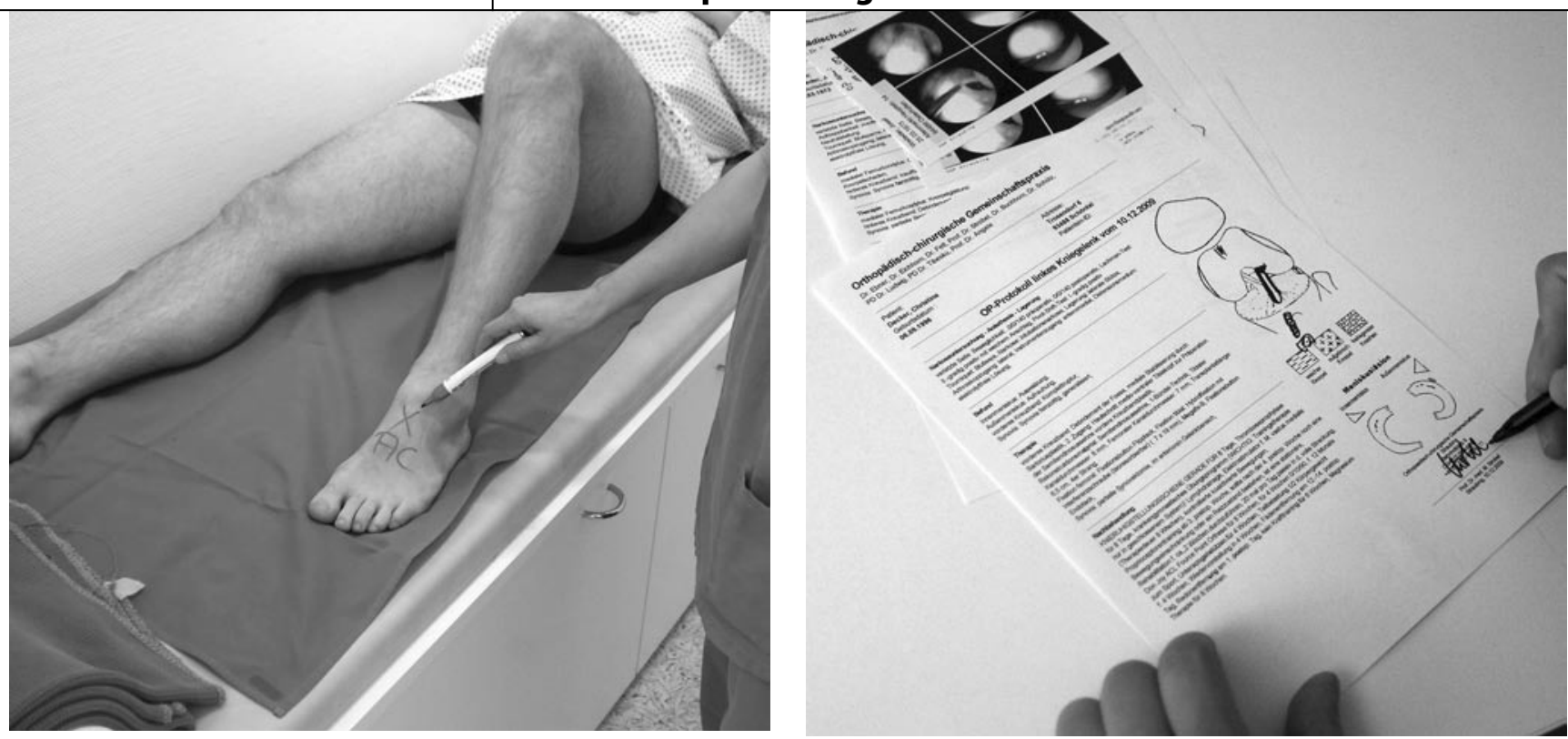

Abb. $1 \Delta$ Markierung des zu operierenden Beins

Abb. $2 \Delta$ Erstellung des Operationsprotokolls unmittelbar nach dem Eingriff

den zuweisenden Orthopäden bzw. Chirurgen aufzusuchen, um sich die Wundfäden entfernen zu lassen. Sind bis zu diesem Zeitpunkt Komplikationen aufgetreten, können diese erkannt und adäquat behandelt werden.

\section{Dokumentationsbereich/Aufwachraum/} Patienteninformation. Nach Abschluss der Operation wird der Patient in den Aufwachraum verbracht. Gleichzeitig erfolgt die Dokumentation der erhobenen arthroskopischen Befunde sowie der durchgeführten operativen Maßnahmen. Bewährt hat sich bei uns ein computerunterstütztes Dokumentationssystem zur Erstellung dieser Operationsprotokolle (AI$\mathrm{DA}^{\oplus}$ ORTHO, Fa. Karl Storz, Tuttlingen), in welchem zudem dokumentiert wird, wie die Nachbehandlung zu erfolgen hat (- Abb. 2).

Im Aufwachraum erhält der Patient nach Erreichen eines ausreichenden Vigilanzniveaus eine erste leichte Mahlzeit sowie ein Getränk. Abschließend wird der Verband auf Intaktheit bzw. auf ein Durchbluten untersucht. Der Patient wird zudem vom Operateur über die durchgeführten operativen Maßnahmen und die erforderliche Nachbehandlung informiert (• Abb. 3).

Entlassung und Ausschleusung. Der Patient wird ausgeschleust und seinen Angehörigen übergeben ( $\bullet$ Abb. 4). Hierbei findet noch einmal eine Information der Angehörigen statt, wie die weitere Nachbehandlung hinsichtlich der Thromboseprophylaxe zu erfolgen hat und dass der Patient am 1. postoperativen Tag seinen Hausarzt bzw. Orthopäden/Chirurgen aufsuchen soll.

\section{Fehler bei ambulanten Operationen}

Einer der häufigsten Fehler bei ambulanten arthroskopischen Operationen liegt nicht in der operativen Durchführung der einzelnen Operationsschritte begründet, sondern in der Indikationsstellung und den daraus resultierenden Folgen. Nicht selten erfolgt eine ambulante Arthroskopie im Sinne einer „diagnostischen Arthroskopie" nach akuter Verletzung. Hierbei wird die schon klinisch vordiagnostizierte Läsion - oft eine VKBRuptur (VKB: vorderes Kreuzband) - diagnostisch gesichert. Diagnostische Arthroskopien weisen aber zahlreiche potenzielle Risiken wie die Läsion des Hoffa-Fettkörpers oder Induktion einer Kapselfibrose auf. Werden Kreuzbandstümpfe reseziert, erschwert dies die Orientierung beim definitiven Kreuzbandersatz. Zudem werden nicht selten elongierte Kreuzbandfasern reseziert, an welchen sich Narbenstrukturen orientieren könnten, um eine Narbenheilung nach Partialruptur zu gewährleisten. Nicht zuletzt ist das Ausspü- len des Hämarthros zeitaufwändig. Der große Nachteil besteht aber darin, dass keine wirklichen therapeutischen Konsequenzen gezogen werden. Daher muss eine Akutarthroskopie seltenen Ausnahmen vorbehalten bleiben.

Wesentlich ist, dass beim ambulanten Eingriff nicht nur der intraartikuläre Schaden festgestellt wird, sondern der definitive Eingriff in gleicher Sitzung erfolgt. Es darf nicht dazu kommen, dass zunächst mit der Arthroskopie die Diagnose gesichert wird und z. B. die Meniskusrefixation dann im Krankenhaus unter stationären Bedingungen durchgeführt werden muss. Eine derartige Indikationsstellung zur ambulanten Arthroskopie ist streng abzulehnen.

\section{Entscheidungskriterien}

Ob ein Patient zur ambulanten Operation geeignet ist bzw. die Indikation zum ambulanten Vorgehen gestellt wird, hängt von verschiedenen Faktoren ab, von denen einige leicht zu erfassen (z. B. Alter), andere dagegen kaum zu beurteilen sind (soziale Umstände, Wohnsituation). Daher muss bei jedem Patienten individuell abgewogen werden, ob ein ambulanter Eingriff möglich ist. 


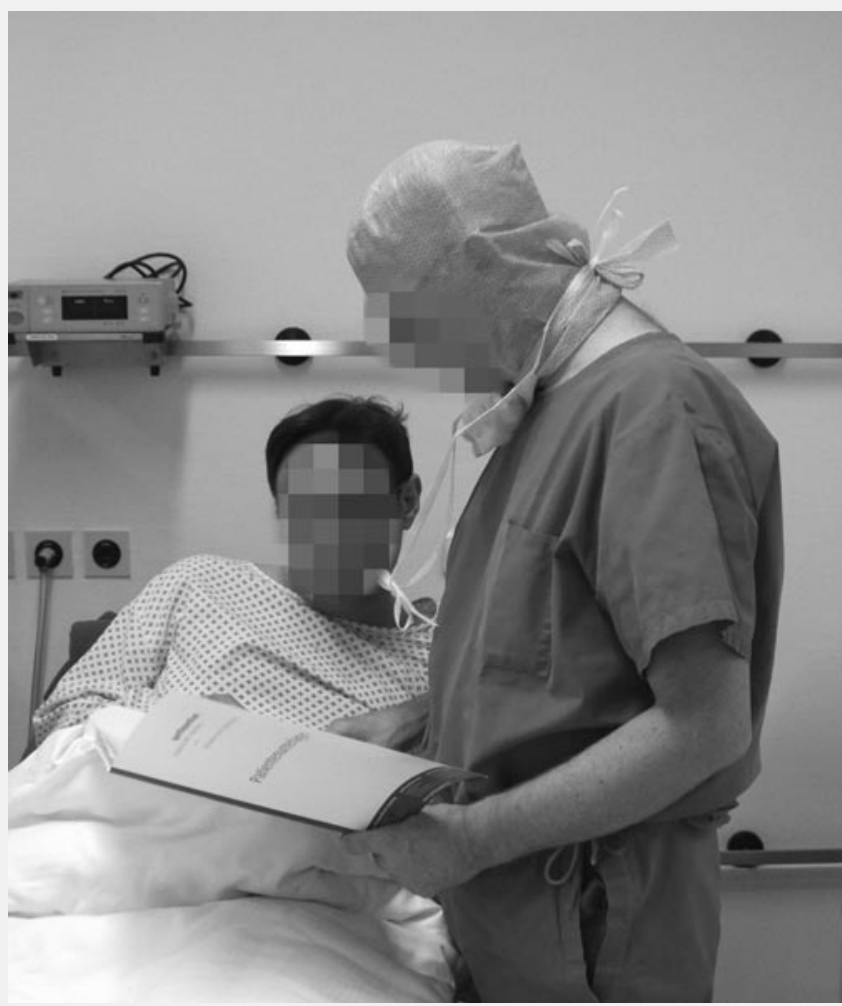

Abb. $3 \Delta$ Informationsgespräch des Operateurs mit dem Patienten über Operationsverlauf und die erforderlichen Nachbehandlungsmaßnahmen

Abb. 4 "In Empfangnahme" des Patienten von seiner Ehefrau

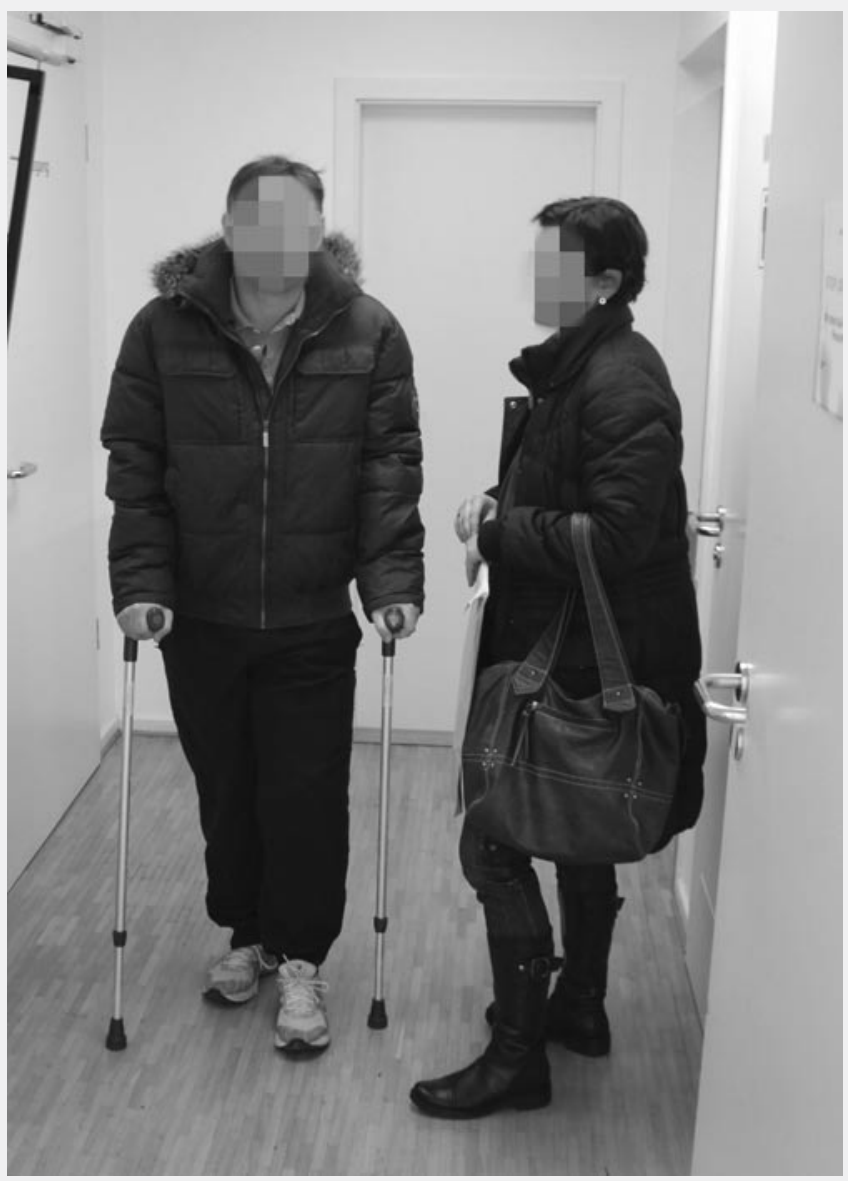

\section{Alter}

Für ambulante Operationen sind sowohl Kinder als auch ältere Patienten - der älteste Patient in unserem Ambulatorium war über 90 Jahre alt - geeignet. Das Alter stellt demnach u. E. keine Kontraindikation zum ambulanten Vorgehen dar, sofern die anderen Parameter (s. unten) dieser Entscheidung nicht entgegenstehen.

\section{Soziales Umfeld}

Die soziale Situation des Patienten ist ein ganz entscheidender Parameter, ob dieser für das ambulante Vorgehen geeignet ist oder ob er stationär operiert werden sollte. Lebt ein Patient allein und/oder ist er tagsüber nach dem Eingriff allein in seiner Wohnung, stellt dies u. E. eine strenge Kontraindikation für einen ambulanten Eingriff dar. Kostenträger sehen diese Situation gerne anders. Dennoch ist nicht abzustreiten, dass wesentlich höhere Folgekosten entstehen können, wenn bei einem derart unbeobachteten bzw. unbe- aufsichtigten Patienten Komplikationen auftreten.

\section{Wohnsituation}

Wohnt der Patient im 3., 4. oder 5. Stock und besitzt das Haus keinen Fahrstuhl, ist dies als gewisse Kontraindikation zum ambulanten Eingriff anzusehen. Gleiches gilt, wenn sich die Toilette oder das Badezimmer 1 oder 2 Etagen entfernt vom Schlafzimmer bzw. dem Zimmer befinden, wo sich der Patient meistens aufhält.

\section{Begleiterkrankungen}

Gravierende Begleiterkrankungen wie Hypertonie, Adipositas per magna, Diabetes mellitus, vorangegangene Komplikationen nach ambulanten Eingriffen sollten sehr sorgfältig evaluiert werden, da sie eine Kontraindikation für den ambulanten Eingriff darstellen können. Im Zweifelsfall sollte das stationäre Vorgehen bevorzugt werden.

\section{Entfernung Ambulantes Operationszentrum - Wohnung}

Befindet sich das ambulante Operationszentrum nur wenige Kilometer vom Heimatort des Patienten entfernt, stellt dies kein Problem dar. Aufgrund der zunehmenden Spezialisierung, auch im arthroskopischen Bereich, suchen Patienten gerne Operateure auf, die umfangreiche Erfahrungen in der arthroskopischen Chirurgie besitzen und Eingriffe auf hohem Niveau ambulant durchführen. Dies erfordert manchmal eine längere Anreise zum Operationsort. Beträgt diese mehr als $200 \mathrm{~km}$, sollte überlegt werden, ob der Patient nicht eine Nacht in der Nähe des ambulanten Operationszentrums verbringen oder in diesem selbst übernachten kann, sofern eine größere arthroskopische Operation erfolgte.

Nach Kreuzbandoperationen empfiehlt es sich generell, den Patienten in der ersten Nacht engmaschig zu überwachen. Da bei uns die Patienten oft mehrere hundert Kilometer weit anreisen, hat es 
sich bewährt, dass sie die erste Nacht im ambulanten Operationszentrum verbringen. Hierdurch entstehen den Kostenträgern keine Mehrkosten, es ist jedoch gesichert, dass es im unmittelbar postoperativen Verlauf nicht zu gravierenden Komplikationen wie Nachblutungen, überschießenden Schmerzreaktionen oder sonstigen Störungen kommt.

\section{Kosten}

Sie sollten eigentlich kein Kriterium darstellen, nachdem die Indikation zwischen einer ambulanten und einer stationären Vorgehensweise abgewogen wurde. Dennoch sehen sich viele Kostenträger bemüBigt, den Patienten unbedingt zu einer ambulanten Operation zu drängen, da diese die kostengünstigere Therapieform darstellt. Bei der „ärztlichen Indikationsstellung" zum ambulanten Eingriff sollten der Kostenfaktor oder der Druck des Kostenträgers auf den Operateur und den $\mathrm{Pa}$ tienten keine oder nur eine sehr untergeordnete Rolle spielen.

\section{Patientenwunsch}

Patienten, die schon einmal ambulant operiert wurden, stehen dieser Eingriffsart entweder sehr positiv gegenüber oder lehnen sie kategorisch ab. Hat ein Patient einmal starke Schmerzen und/oder Unwohlsein nach einem ambulanten operativen Eingriff erlebt, lehnt er einen erneuten ambulanten Eingriff ab.

Andere Patienten wollen dagegen wieder sehr schnell fit und aktiv sein, wenn möglich noch am Tag der Operation wieder ihre berufliche Tätigkeit aufnehmen. Auch bei dieser Patientengruppe gilt es, die Indikation zum ambulanten Eingriff sehr kritisch zu überprüfen. Es ist nicht sinnvoll, dass der Patient nach einer ambulanten Operation - auch wenn nur 2 kleine Hautinzisionen am Kniegelenk erfolgten - seine normale berufliche Tätigkeit, z. B. eine Schreibtischtätigkeit, sofort wieder aufnimmt. Man muss bedenken, dass auch eine ambulante partielle oder subtotale Meniskusresektion zu einem gravierenden Druckanstieg im Bereich des betroffenen Gelenkkompartments führt. Das Gelenk muss sich an diesen $\mathrm{Zu}$ stand erst gewöhnen. Wird es zu schnell belastet, können rezidivierende Reizergüsse und chronische Schmerzen resultieren, die den weiteren Behandlungsverlauf prägen und sich über mehrere Wochen, manchmal Monate erstrecken können.

\section{Erkrankung - Verletzung}

Die Indikation zum operativen Eingriff sollte in Abhängigkeit der Diagnose gestellt werden, jeweils unter Abwägung der oben genannten Parameter. Nicht alle Eingriffe können und sollten ambulant durchgeführt werden. Hier gilt es insbesondere, zu berücksichtigen, ob das erforderliche Instrumentarium in ausreichender Menge und in hinreichender Differenziertheit vorhanden ist.

Theoretisch kann jede Knieoperation ambulant erfolgen. Gut und sicher sind

- sämtliche Arten von Meniskusoperationen,

- Knorpeleingriffe,

- Synoviaeingriffe,

- Eingriffe bei Patellaluxationen sowie

- Kreuzbandoperationen

ambulant durchführbar. Entscheidend ist, dass der Operateur den Eingriff beherrscht und das erforderliche Operationsinstrumentarium in ausreichender Menge vorhanden ist. Falls ein Instrument hinunterfällt oder unsteril wird, muss immer ein Ersatzinstrument vorhanden sein. Für verschiedene Eingriffe ist eine Durchleuchtungsvorrichtung erforderlich. In einigen ambulanten Operationszentren ist dies nicht gewährleistet, sodass derartige Eingriffe dann dem stationären Vorgehen vorbehalten bleiben.

Ambulant können vordere und sogar isolierte hintere Kreuzbandrekonstruktionen durchgeführt werden. Bei Revisionsoperationen, insbesondere aber kombinierten Rekonstruktionen mit Ersatz eines Kreuzbandes und einer peripheren Bandstruktur, sollte unbedingt ein stationäres Vorgehen bevorzugt werden, da oft von der Gegenseite Sehnen für die Transplantate entnommen werden müssen. Eine beidseitige Sehnenentnahme aber ist unter ambulanten Bedingungen nicht sinnvoll. Auch Revisionsoperationen nach primärem Kreuzbandersatz sollten dem stationären Vorgehen vorbehalten bleiben.
Bei folgenden Eingriffen ist die ambulante Durchführung nicht sinnvoll:

- Kreuzbandrevision,

- Frakturversorgung,

- komplexe Rekonstruktionen mit Rekonstruktion eines Kreuzbandes und peripherer Strukturen bzw. Transplantatentnahme von der kontralateralen Seite,

- offene Knorpeltransplantation,

- Infektion und

- Spongiosaplastiken mit Spongiosaentnahme aus dem Beckenkamm.

\section{Kontraindikation für ambulante Arthroskopie}

Hier sind anzuführen:

- Blutungsanamnese,

- Dialyse,

- ASA 3 und 4 (ASA: American Society of Anesthesiologists, kardiale Begleiterkrankungen),

- fehlende häusliche postoperative Versorgung z. B. Selbstversorger, allein lebender Student am Studienort usw., - unzumutbare Wohnsituation (z. B. 4. Stock ohne Aufzug),

- Entfernung zum Wohnort mehr als 30 oder $50 \mathrm{~km}$ ohne alternative medizinische Versorgungsmöglichkeit vor Ort und

- Eingriffe mit unkalkulierbarer postoperativer Schmerzsituation.

\section{Diskussion}

Arthroskopien zählen zu den häufigsten ambulant durchgeführten Eingriffen. Am Knie sind prinzipiell alle arthroskopischen Eingriffe ambulant durchführbar. Je umfangreicher jedoch das Operationsspektrum, desto höher ist auch der instrumentelle und personelle Aufwand für das ambulante Operationszentrum und den Operateur.

Das ambulante Operieren erfordert ein exaktes und wohlorganisiertes prä-, intraund postoperatives Management, um effizient zum Wohle des Patienten, aber auch gleichzeitig kostenorientiert den Eingriff ablaufen zu lassen. Bei größeren ambulanten Operationen sollten der Operateur bzw. das Operationszentrum für die Nachsorge sorgen. Eine Übernachtungsmöglichkeit im oder nahe dem Operati- 
onszentrum sollte möglich sein, sodass die Patienten nicht postoperativ einen langen Heimweg antreten müssen. Durch die differenzierte Patientenselektion ist das Komplikationsrisiko deutlich zu reduzieren.

Insbesondere gilt es zu beachten, dass nur dann operiert werden sollte, wenn der Operateur den jeweiligen Eingriff perfekt beherrscht. Die ambulante Arthroskopie darf nicht dazu führen, dass nur die klinische Diagnose bestätigt wird, therapeutische Maßnahmen aber nicht erfolgen, sodass sich der Patient einem erneuten operativen Eingriff unter stationären Bedingungen unterziehen muss. Die rein diagnostische Arthroskopie ist zu vermeiden, dies gilt sowohl für den stationären als auch insbesondere für den ambulanten Bereich. Sinnvoll sind dagegen Staging-Arthroskopien z. B. nach fehlgeschlagenem vorderem Kreuzbandersatz oder zum Ausschluss peripherer Instabilitäten. Derartige Eingriffe können ambulant durchgeführt werden.

\section{Fazit}

Ambulante Arthroskopien sind dann erfolgreich, wenn nicht nur die Auflauforganisation stimmig ist, sondern auch eine differenzierte Patientenselektion erfolgt. Bei der Indikationsstellung zum ambulanten Eingriff ist insbesondere das soziale Umfeld des Patienten zu berücksichtigen. Postoperativ muss die Nachsorge so gesichert sein, dass der Patient in den ersten $\mathbf{2 4} \mathrm{h}$ nie alleine in seiner Wohnung ist. Ein Verwandter, Lebenspartner oder enger Freund sollte permanent anwesend sein. Ist dies nicht gewährleistet oder kann der Patient dies nicht zusichern, stellt das eine eindeutige Kontraindikation für das ambulante Vorgehen dar. In einem derartigen Fall muss dem Patienten das stationäre Vorgehen nahegelegt werden. Wünsche bzw. Forderungen des Kostenträgers nach einem ambulanten Eingriff sind bei der Entscheidungsfindung sekundär, schließlich obliegt dem Operateur die Verantwortung für den intra- und auch postoperativen Verlauf.
Korrespondenzadresse

Prof. Dr. M.J. Strobel

Sporthopaedicum Straubing, Bahnhofplatz 8, 94315 Straubing

strobel@sporthopaedicum.de

Interessenkonflikt. Keine Angaben

\section{Literatur}

Beim Verfasser 